\title{
Essential Norm of Operators into Weighted-Type Spaces on the Unit Ball
}

\author{
Pablo Galindo, ${ }^{1}$ Mikael Lindström, ${ }^{2}$ and Stevo Stevi ${ }^{3}$ \\ ${ }^{1}$ Departamento de Análisis Matemático, Universidad de Valencia, 46.100 Burjasot, Valencia, Spain \\ ${ }^{2}$ Department of Mathematical Sciences, University of Oulu, 90014 Oulu, Finland \\ ${ }^{3}$ Mathematical Institute of the Serbian Academy of Sciences, Knez Mihailova 36/III, 11000 Beograd, Serbia \\ Correspondence should be addressed to Stevo Stević, sstevic@ptt.rs
}

Received 13 December 2010; Revised 8 May 2011; Accepted 10 May 2011

Academic Editor: Nikolaos Papageorgiou

Copyright (C) 2011 Pablo Galindo et al. This is an open access article distributed under the Creative Commons Attribution License, which permits unrestricted use, distribution, and reproduction in any medium, provided the original work is properly cited.

The essential norm of any operator from a general Banach space of holomorphic functions on the unit ball in $\mathbb{C}^{n}$ into the little weighted-type space is calculated. Some applications of the formula are given.

\section{Introduction and Preliminaries}

Characterizing the compactness of composition or weighted composition operators, their differences, and Toeplitz operators between Banach spaces of analytic functions has attracted attention of numerous authors, and there has been a great interest in the matter of calculating or estimating essential norms of operators, see, for example, [1-8]. Motivated by this line of investigations, the first two authors calculated in [3] the essential norm of any operator acting between weighted-type spaces or between Bloch spaces on the unit disk and also estimated it on the weighted-Bergman space $A_{\alpha}^{1}(\mathbb{D})$.

We obtain a formula for the essential norm of any operator into a weighted-type space on the unit ball in $\mathbb{C}^{n}$ whose domain space belongs to a general class of Banach holomorphic function spaces, thus extending to the case of the unit ball some results in [3]. Some applications of our main results are given.

Let $\mathbb{B}$ be the open unit ball in the euclidian complex-vector space $\mathbb{C}^{n}$ and $H(\mathbb{B})$ the space of all holomorphic functions on $\mathbb{B}$. The pseudohyperbolic distance between $z, u \in \mathbb{B}$ is denoted by $\rho(z, u)$ (see [9] for more details). If $X$ is a Banach space, by $B_{X}$ we denote the closed unit ball in $X$. 
Let $v$ be a positive continuous function on $\mathbb{B}$ (weight). The weighted-type space (or Bergman space of infinite order) $H_{v}^{\infty}(\mathbb{B})=H_{v}^{\infty}$ is defined by

$$
H_{v}^{\infty}=\left\{f \in H(\mathbb{B}):\|f\|_{H_{v}^{\infty}}:=\sup _{z \in \mathbb{B}} v(z)|f(z)|<\infty\right\} .
$$

The little weighted-type space $H_{v}^{0}(\mathbb{B})=H_{v}^{0}$ consists of all $f \in H_{v}^{\infty}$ such that

$$
\lim _{|z| \rightarrow 1} v(z)|f(z)|=0
$$

With the norm $\|\cdot\|_{H_{v}^{\infty}}$, both are Banach spaces. The norm topology of $H_{v}^{\infty}$ is finer than the topology $\tau$ of uniform convergence on compact subsets of $\mathbb{B}$. For $v_{\alpha}(z)=\left(1-|z|^{2}\right)^{\alpha}, \alpha>0$, the standard weighted-type spaces, which we denote by $H_{\alpha}^{\infty}$ and $H_{\alpha}^{0}$, are obtained. These spaces appear in the study of growth conditions of analytic functions, see, for example, [10, 11].

The Bloch-type space $\mathbb{B}_{\mu}(\mathbb{B})=\mathbb{B}_{\mu}$ consists of all $f \in H(\mathbb{B})$ such that

$$
b_{\mu}(f)=\sup _{z \in \mathbb{B}} \mu(z)|\Re f(z)|<\infty,
$$

where $\mu$ is a weight,

$$
\Re f(z)=\sum_{j=1}^{n} z_{j} \frac{\partial f}{\partial z_{j}}(z)=\langle\nabla f(z), \bar{z}\rangle
$$

is the radial derivative of $f$, and $\nabla f$ is the complex gradient of $f$.

The little Bloch-type space $\mathbb{B}_{\mu, 0}(\mathbb{B})=\mathbb{B}_{\mu, 0}$ consists of all $f \in \mathbb{B}_{\mu}$ such that

$$
\lim _{|z| \rightarrow 1} \mu(z)|\Re f(z)|=0 .
$$

Both are Banach spaces with the norm $\|f\|_{\mathcal{B}_{\mu}}=|f(0)|+b_{\mu}(f)$. For the standard weight $v_{p}$, $p>0$, we get the $p$-Bloch space $\mathbb{B}^{p}$ and the little $p$-Bloch space $\boldsymbol{B}_{0}^{p}$.

The standard weighted-Bergman space $A_{\beta}^{p}(\mathbb{B})=A_{\beta}^{p}, \beta>-1, p \geq 1$, is the set of all analytic functions on $\mathbb{B}$ such that

$$
\|f\|_{A_{\beta}^{p}}^{p}=\int_{\mathbb{B}}|f(z)|^{p} c_{\beta}\left(1-|z|^{2}\right)^{\beta} d V(z)<\infty,
$$

where $d V(z)$ is the normalized volume measure on $\mathbb{B}$ and $c_{\beta}=\Gamma(n+\beta+1) / n ! \Gamma(\beta+1)$.

A weight $v$ is radial if it satisfies $v(z)=v(|z|)$ for every $z \in \mathbb{B}$. Throughout this paper we assume that all weights $v$ are typical, that is, they are radial, nonincreasing with respect to $|z|$ and such that $\lim _{|z| \rightarrow 1^{-}} v(z)=0$. Many results on weighted-type spaces of analytic functions and on operators between them are given in terms of the so-called associated weights 
(see [10]) and in terms of the weights. For a weight $v$ the associated weight $\widetilde{v}$ is defined as follows

$$
\widetilde{v}(z):=\left(\sup \left\{|f(z)|: f \in H_{v}^{\infty},\|f\|_{H_{v}^{\infty}} \leq 1\right\}\right)^{-1}
$$

For a typical weight $v$ the associated weight $\tilde{v}$ is also typical. Furthermore, for each $z \in \mathbb{B}$ there is an $f_{z} \in H_{v}^{\infty},\left\|f_{z}\right\|_{H_{v}^{\infty}} \leq 1$, such that $f_{z}(z)=1 / \widetilde{v}(z)$, and the same holds for the space $H_{v}^{0}$. It is known that $H_{\tilde{v}}^{\infty} \cong H_{v}^{\infty}$ and $H_{\tilde{v}}^{0} \cong H_{v}^{0}$, that is, they are isometrically isometric [10].

We say that a weight $v$ satisfies condition $(L 1)$ if it is radial and

$$
\inf _{k \in \mathbb{N}} \frac{v\left(1-2^{-(k+1)}\right)}{v\left(1-2^{-k}\right)}>0
$$

For radial weights $v$ satisfying condition $(L 1)$, we have that $v$ and $\widetilde{v}$ are equivalent, that is, there is a $C \geq 1$ such that $v \leq \widetilde{v} \leq C v$. Recently Lusky and Taskinen [12] have shown, among other results, that $H_{\alpha}^{0}$ is isomorphic to $c_{0}$.

Since the closed unit ball $B_{H_{v}^{\infty}}$ is a compact subset of $(H(\mathbb{B}), \tau)$, a result of Dixmier-Ng [13] gives that the subspace of $\left(H_{v}^{\infty}\right)^{*}$

$$
G_{v}^{\infty}:=\left\{l \in\left(H_{v}^{\infty}\right)^{*}: l \mid B_{H_{v}^{\infty}} \text { is } \tau \text {-continuous }\right\}
$$

is a predual of $H_{v}^{\infty}$, that is, $\left(G_{v}^{\infty}\right)^{*} \cong H_{v}^{\infty}$. Clearly the evaluation functional at $z \in \mathbb{B}$, defined by $\delta_{z}(f)=f(z)$, belongs to $G_{v}^{\infty}$. The norm of $\delta_{z}$ is denoted by $\left\|\delta_{z}\right\|_{v}$. Moreover, the set $\left\{\delta_{z}\right.$ : $z \in \mathbb{B}\}$ is a total set, that is, its linear span is norm dense in $G_{v}^{\infty}$. More precisely, the next isomorphism result is due to Bierstedt and Summers.

Lemma 1.1 (see [11]). The map $f \mapsto[l \mapsto\langle l, f\rangle]$ is an onto isometric isomorphism between $H_{v}^{\infty}$ and $\left(G_{v}^{\infty}\right)^{*}$ and the restriction map $\left.l \mapsto l\right|_{H_{v}^{0}}$ gives rise to an isometric isomorphism between $G_{v}^{\infty}$ and $\left(H_{v}^{0}\right)^{*}$. following.

Similarly to the corresponding result in the one variable [14], one can prove the

Lemma 1.2. Suppose $\beta>-1$ and $\gamma>0$. Then $\left(A_{\beta}^{1}\right)^{*}$ is isomorphic to $H_{\gamma}^{0}$

$$
\langle f, g\rangle_{\beta, \gamma}=\int_{\mathbb{B}} f(z) \overline{g(z)} c_{\beta+\gamma} v_{\beta+\gamma}(z) d V(z), \quad f \in H_{\gamma}^{\infty}, g \in A_{\beta}^{1} .
$$

Moreover, under the pairing $\langle f, g\rangle_{\beta, \gamma}$ with $g \in H_{\gamma}^{0}$ and $f \in A_{\beta}^{1}$, we also have that $\left(H_{\gamma}^{0}\right)^{*}$ is isomorphic to $A_{\beta}^{1}$.

$$
\text { For } z, w \in \mathbb{B} \text {, let }
$$

$$
K_{z}^{\beta}(w)=\frac{1}{(1-\langle w, z\rangle)^{n+\beta+1}} .
$$


Then the kernel function $K_{z}^{\beta+\gamma}$ clearly belongs to $H_{\gamma}^{0}$ and to $A_{\beta}^{1}$. It has the reproducing property

$$
f(z)=\int_{\mathbb{B}} f(w) \overline{K_{z}^{\beta}(w)} c_{\beta} v_{\beta}(w) d V(w), \quad z \in \mathbb{B},
$$

for every function $f \in A_{\beta}^{1}$ (see [9, Theorem 2.2]). As a direct application we get that $g(w)=$ $\left\langle K_{w}^{\beta+\gamma}, g\right\rangle_{\beta, \gamma}$ for all $g \in A_{\beta}^{1}$ and $f(w)=\left\langle f, K_{w}^{\beta+\gamma}\right\rangle_{\beta, \gamma}$ for all $f \in H_{\gamma}^{\infty}$.

Let $E$ be a Banach space of analytic functions on $\mathbb{B}$ containing the constant functions. We denote its norm by $\|\cdot\|_{E}$. With $\|\cdot\|_{E^{*}}$ we denote the norm of its Banach dual space $E^{*}$. Consider the following conditions on $E$.

(C1) There are positive constants $s$ and $C$ such that

$$
|f(z)| \leq C \frac{\|f\|_{E}}{\left(1-|z|^{2}\right)^{s}}, \quad \text { for every } f \in E \text {, and for each } z \in \mathbb{B} \text {. }
$$

(C2) The analytic polynomials are dense on $E$.

(C3) $\left\|\delta_{z}\right\|_{E^{*}} \rightarrow \infty$, when $|z| \rightarrow 1$.

(C4) The linear span of the set $\left\{\delta_{z}: z \in \mathbb{B}\right\}$ is dense in $E^{*}$.

(C5) There is a $C>0$ such that $\|f\|_{E} \leq C\|f\|_{\infty}$ for all $f \in E$, where $\|f\|_{\infty}=\sup _{z \in \mathbb{B}}|f(z)|$, the supremum taken in the extended real line.

It follows from $(C 1)$ that the closed unit ball $B_{E}$ is $\tau$-bounded, hence it is an equicontinuous and a $\tau$-relatively compact set. Moreover, we have the following.

Proposition 1.3. Assume that E satisfies conditions (C1) and (C5). Then the contraction operators $K_{r}: E \rightarrow$ E given by

$$
K_{r}(f)(z):=f(r z), \quad 0<r<1,
$$

are well defined and compact.

Proof. Each given $f \in E$ may be approximated uniformly on compact subsets by the sequence $\left(S_{k}\right)_{k \in \mathbb{N}_{0}}$ of its Taylor polynomials at 0 . Therefore, $S_{k}(r z) \rightarrow f(r z)$ uniformly on $\mathbb{B}$ as $k \rightarrow \infty$. Further, $\left(S_{k}(r z)\right)_{k \in \mathbb{N}_{0}} \subset E$ is a Cauchy sequence by $(C 5)$, so it converges to some element in $E$. Hence $K_{r}(f) \in E$.

Moreover $K_{r}$ is compact. Indeed, any sequence $\left(f_{m}\right)_{m \in \mathbb{N}} \subset B_{E}$ has a $\tau$-convergent subsequence, say itself, to an element $g \in H(\mathbb{B})$. Therefore $\left(f_{m}(r z)\right)_{m \in \mathbb{N}}$ converges uniformly on $\mathbb{B}$ to $g(r z)$, and again $(C 5)$ yields that $\left(K_{r}\left(f_{m}\right)\right)_{m \in \mathbb{N}}$ is a Cauchy sequence in $E$ that converges to $K_{r}(g)$.

We consider another condition on $E$.

(C6) For every $f \in E$, we have $\left\|K_{r}(f)\right\|_{E} \leq\|f\|_{E}$ for $0<r<1$. 
Remarks. (a) Note that (C6) implies that $\left\|K_{r}\right\|_{E \rightarrow E}=\sup _{\|f\|_{E} \leq 1}\left\|K_{r}(f)\right\|_{E} \leq 1$.

(b) By (C1) every functional at $z \in \mathbb{B}_{n}, \delta_{z}: E \rightarrow \mathbb{C}$, is bounded, and therefore

$$
\left|\delta_{z}(f)\right| \leq\left\|\delta_{z}\right\|_{E^{*}}\|f\|_{E^{\prime}} \quad \text { for every } f \in E .
$$

(c) Spaces like $H_{v}^{0}$, the little Bloch space, Hardy spaces $H^{p}$ and weighted-Bergman spaces $A_{\beta}^{p}$ fulfill conditions $(C 1)-(C 6)$. For $H^{p}, s=n / p$, and for $A_{\beta}^{p}$, we have that $s=(n+\beta+$ $1) / p$ (see [9]).

Let $X$ and $Y$ be Banach spaces. The essential norm of a bounded operator $T$ is the distance in the operator norm from $T$ to the compact operators, that is,

$$
\|T\|_{e}=\inf \left\{\|T+K\|_{X \rightarrow Y}: K \text { is compact }\right\} .
$$

We write $A \leq B$ if there is a positive constant $C$, not depending on properties of $A$ and $B$, such that $A \leq C B$. We also write $A \simeq B$ whenever $A \leq B$ and $B \leq A$.

\section{The Essential Norm of Operators}

The next proposition is an extension of Proposition 2.1 in [5].

Proposition 2.1. Assume that E satisfies conditions (C1)-(C6). Then there exists a sequence $\left(L_{m}\right)_{m \in \mathbb{N}}$ of compact operators on E such that

(i) for any $0<t<1$,

$$
\lim _{m \rightarrow \infty} \sup _{\|f\|_{E} \leq 1} \sup _{|z| \leq t}\left|\left(I-L_{m}\right)(f)(z)\right|=0
$$

(ii)

$$
\limsup _{m \rightarrow \infty}\left\|I-L_{m}\right\|_{E \rightarrow E} \leq 1
$$

Proof. We prove that for every $0<t<1$ and $\varepsilon>0$ there is a compact operator $L: E \rightarrow E$ such that

$$
\|I-L\|_{E \rightarrow E}<1+2 \varepsilon,
$$

and

$$
\sup _{\|f\|_{E} \leq 1} \sup _{|z| \leq t}|(I-L) f(z)|<\varepsilon .
$$

When this is done, a standard diagonal argument by taking a sequence $\left(t_{n}\right) \uparrow 1$ and a sequence of positive numbers $\left(\varepsilon_{n}\right) \downarrow 0$ will give the result. 
The operator $L$ will be constructed as a suitable finite convex combination of the operators $K_{r}$ and therefore by Proposition 1.3, it will be compact.

The operators $I-K_{r}:(H(\mathbb{B}), \tau) \rightarrow(H(\mathbb{B}), \tau)$ are continuous, and for each $f \in H(\mathbb{B})$, we have that $\left(I-K_{r}\right) f \rightarrow 0$ in $(H(\mathbb{B}), \tau)$ when $r \rightarrow 1$. By the Banach-Steinhaus theorem for Fréchet spaces $\left(I-K_{r}\right) \rightarrow 0$ uniformly on relatively compact subsets of $(H(\mathbb{B}), \tau)$ as $r \rightarrow 1$; thus in particular on $B_{E}$. Hence, if we pick $s_{1}$ such that $t<s_{1}<1$, we have

$$
\lim _{r \rightarrow 1} \sup _{\|f\|_{E^{1}} \leq 1|z| \leq s_{1}} \sup _{1}\left|\left(I-K_{r}\right) f(z)\right|=0
$$

Since $B_{E}$ is an equicontinuous set, the operator $\delta: \mathbb{B} \rightarrow E^{*}, z \mapsto \delta_{z}$ is continuous, so we can find $z_{0} \in \mathbb{B}$ such that $\sup _{|z| \leq s_{1}}\left\|\delta_{z}\right\|_{E^{*}}=\left\|\delta_{z_{0}}\right\|_{E^{*}}$. Moreover we have the inequalities

$$
\frac{1}{\|1\|_{E}}=\frac{\left|\delta_{z}(1)\right|}{\|1\|_{E}} \leq\left\|\delta_{z}\right\|_{E^{*}}
$$

Therefore, we find an $r_{1} \in(0,1)$ such that

$$
\sup _{\|f\|_{E} \leq 1} \sup _{|z| \leq s_{1}} \frac{\left|\left(I-K_{r_{1}}\right) f(z)\right|}{\left\|\delta_{z}\right\|_{E^{*}}}<\min \left\{\varepsilon, \frac{\varepsilon}{\left\|\delta_{z_{0}}\right\|_{E^{*}}}\right\}
$$

Since the bounded set $\left\{\delta_{z} /\left\|\delta_{z}\right\|_{E^{*}}: z \in \mathbb{B}\right\}$ is equicontinuous in $E^{*}$, the weak*topology coincides on it with the coarser one of the convergence on the dense subset of the polynomials, and also with the finer one of the uniform convergence on relatively compact sets in $E$. Moreover, observe that for each polynomial $P$,

$$
\lim _{|z| \rightarrow 1}\left\langle\frac{\delta_{z}}{\left\|\delta_{z}\right\|_{E^{*}}}, P\right\rangle=\lim _{|z| \rightarrow 1} \frac{P(z)}{\left\|\delta_{z}\right\|_{E^{*}}}=0
$$

since condition $(C 3)$ holds and $P$ is bounded on $\mathbb{B}$.

This means that $\lim _{|z| \rightarrow 1} \delta_{z} /\left\|\delta_{z}\right\|_{E^{*}}=0$ on relatively compact sets in $E$, in particular on $K_{r_{1}}\left(B_{E}\right)$. Hence there is an $s_{2}>s_{1}$ such that for each $f \in B_{E}$, we have

$$
\sup _{|z| \geq s_{2}} \frac{\left|\delta_{z}\left(K_{r_{1}} f\right)\right|}{\left\|\delta_{z}\right\|_{E^{*}}} \leq \varepsilon
$$

Therefore,

$$
\sup _{\|f\|_{E} \leq 1} \sup _{|z| \geq s_{2}} \frac{\left|\left(I-K_{r_{1}}\right) f(z)\right|}{\left\|\delta_{z}\right\|_{E^{*}}}<1+\varepsilon
$$



satisfying

We can continue in this way and find two strictly increasing sequences $\left(s_{k}\right)$ and $\left(r_{k}\right)$

$$
\begin{aligned}
& \sup _{\|f\|_{E} \leq 1} \sup _{|z| \leq s_{k}} \frac{\left|\left(I-K_{r_{k}}\right) f(z)\right|}{\left\|\delta_{z}\right\|_{E^{*}}}<\min \left\{\varepsilon, \frac{\varepsilon}{\left\|\delta_{z_{0}}\right\|_{E^{*}}}\right\}, \\
& \sup _{\|f\|_{E} \leq 1} \sup _{|z| \geq s_{k+1}} \frac{\left|\left(I-K_{r_{k}}\right) f(z)\right|}{\left\|\delta_{z}\right\|_{E^{*}}}<1+\varepsilon .
\end{aligned}
$$

Let $m>1 / \varepsilon$ and put

$$
L=\frac{1}{m} \sum_{k=1}^{m} K_{r_{k}}
$$

Then by (2.11) and the fact that $\left\|\delta_{z_{0}}\right\|_{E^{*}} \geq\left\|\delta_{z}\right\|_{E^{*}}$ for $|z| \leq s_{1}$, we have that (2.4) holds since

$$
\sup _{\|f\|_{E} \leq 1} \sup _{|z| \leq t}|(I-L) f(z)| \leq \frac{1}{m} \sum_{k=1\|f\|_{E} \leq 1}^{m} \sup _{|z| \leq s_{1}}\left|\left(I-K_{r_{k}}\right) f(z)\right|<\varepsilon .
$$

Now we show that (2.3) holds. Similarly to above, we have that

$$
\sup _{\|f\|_{E} \leq 1} \sup _{|z|<t} \frac{|(I-L) f(z)|}{\left\|\delta_{z}\right\|_{E^{*}}}<\varepsilon .
$$

Moreover, by (2.12), if $s_{l}<|z| \leq s_{l+1}$ and $\|f\|_{E} \leq 1$, then

$$
\frac{\left|\left(I-K_{r_{k}}\right) f(z)\right|}{\left\|\delta_{z}\right\|_{E^{*}}}<1+\varepsilon,
$$

except possibly for $k=l$, in which case

$$
\frac{\left|\left(I-K_{r_{l}}\right) f(z)\right|}{\left\|\delta_{z}\right\|_{E^{*}}} \leq\left\|\left(I-K_{r_{l}}\right) f\right\|_{E} \leq\left\|I-K_{r_{l}}\right\|_{E \rightarrow E} \leq 2 .
$$

Hence, we get for all $z \in \mathbb{B}$ and $\|f\|_{E} \leq 1$ that

$$
\frac{|(I-L) f(z)|}{\left\|\delta_{z}\right\|_{E^{*}}} \leq \frac{1}{m} \sum_{k \neq l} \frac{\left|\left(I-K_{r_{k}}\right) f(z)\right|}{\left\|\delta_{z}\right\|_{E^{*}}}+\frac{1}{m} \frac{\left|\left(I-K_{r_{1}}\right) f(z)\right|}{\left\|\delta_{z}\right\|_{E^{*}}}<1+2 \varepsilon .
$$

In light of condition (C4), we have

$$
\|I-L\|_{E \rightarrow E}=\sup _{z \in \mathbb{B}}\left\|(I-L)^{*} \frac{\delta_{z}}{\left\|\delta_{z}\right\|_{E^{*}}}\right\|_{E^{*}}=\sup _{z \in \mathbb{B}} \sup _{\|f\|_{E} \leq 1} \frac{|(I-L) f(z)|}{\left\|\delta_{z}\right\|_{E^{*}}}<1+2 \varepsilon .
$$

Thus we also have (2.3), and the statement is proved. 
Next we state a formula for the norm of any operator from $E$ to either $H_{v}^{\infty}$ or $H_{v}^{0}$. We omit its proof.

Theorem 2.2. Suppose $T: E \rightarrow H_{v}^{\infty}$ is a bounded operator. Then

$$
\|T\|_{E \rightarrow H_{v}^{\infty}}=\sup _{z \in \mathbb{B}} \frac{\left\|T^{*}\left(\delta_{z}\right)\right\|_{E^{*}}}{\left\|\delta_{z}\right\|_{v}} .
$$

Since many operators can be written as weighted composition operators we state the following useful result. As usual, $u C_{\varphi}(f)=u \cdot(f \circ \varphi)$ where $u$ is an analytic function on $\mathbb{B}$ and $\varphi$ is an analytic self-map of $\mathbb{B}$.

Corollary 2.3. If $u C_{\varphi}: E \rightarrow H_{v}^{\infty}$ is a bounded weighted composition operator, then

$$
\left\|u C_{\varphi}\right\|_{E \rightarrow H_{v}^{\infty}}=\sup _{z \in \mathbb{B}}|u(z)| \widetilde{v}(z)\left\|\delta_{\varphi(z)}\right\|_{E^{*}} .
$$

Example 2.4. Let $E=A_{\beta^{\prime}}^{p} \beta>-1, p>1$ with $1 / p+1 / q=1$ and $\tilde{v}_{\alpha}(z)=v_{\alpha}(z)=\left(1-|z|^{2}\right)^{\alpha}$, $\alpha>0$. Then, since for all $f \in A_{\beta}^{p}$ and $z \in \mathbb{B}$,

$$
|f(z)| \leq \frac{\|f\|_{A_{\beta}^{p}}}{\left(1-|z|^{2}\right)^{(n+1+\beta) / p}}
$$

and since equality is attained at

$$
f_{z}(w)=\frac{\left(1-|z|^{2}\right)^{(n+1+\beta) / p}}{(1-\langle w, z\rangle)^{2(n+1+\beta) / p}}
$$

we get

$$
\left\|\delta_{\varphi(z)}\right\|_{A_{\beta}^{q}}=\frac{1}{\left(1-|\varphi(z)|^{2}\right)^{(n+1+\beta) / p}} .
$$

So we get

$$
\left\|u C_{\varphi}\right\|_{A_{\beta}^{p} \rightarrow H_{\alpha}^{\infty}}=\sup _{z \in \mathbb{B}} \frac{\left(1-|z|^{2}\right)^{\alpha}|u(z)|}{\left(1-|\varphi(z)|^{2}\right)^{(n+1+\beta) / p}}
$$

Now we are ready for our main result. Compare it with Theorem 2.2.

Theorem 2.5. Assume that $E$ satisfies conditions (C1)-(C6). If $T: E \rightarrow H_{v}^{0}$ is a bounded operator, then

$$
\|T\|_{e}=\limsup _{|z| \rightarrow 1^{-}} \frac{\left\|T^{*}\left(\delta_{z}\right)\right\|_{E^{*}}}{\left\|\delta_{z}\right\|_{v}}
$$


Proof. If

$$
\ell:=\limsup _{|z| \rightarrow 1^{-}} \frac{\left\|T^{*}\left(\delta_{z}\right)\right\|_{E^{*}}}{\left\|\delta_{z}\right\|_{v}}
$$

then the same reasoning as in the first part of [3, Theorem 3.1] shows that $\|T\|_{e} \geq \ell$.

To prove the reverse inequality, let $\left(L_{m}\right)_{m \in \mathbb{N}}$ be the sequence provided by Proposition 2.1. Then

$$
\|T\|_{e} \leq\left\|T-T \circ L_{m}\right\|_{E \rightarrow H_{v}^{0}}
$$

for all $m \in \mathbb{N}$.

Next, we estimate

$$
\limsup _{m \rightarrow \infty}\left\|T-T \circ L_{m}\right\|_{E \rightarrow H_{v}^{0}}
$$

Fix $0<t<1$ and $f$ in the unit ball of $E$. Then

$$
\left|\left[T \circ\left(I-L_{m}\right)\right](f)(z)\right|=\left|\left\langle\delta_{z},\left[T \circ\left(I-L_{m}\right)\right](f)\right\rangle\right|=\left|\left\langle\left(I-L_{m}^{*}\right) \circ T^{*}\left(\delta_{z}\right), f\right\rangle\right| .
$$

The sequence $\left(I-L_{m}^{*}\right)_{m \in \mathbb{N}} \subset \mathcal{L}\left(E^{*}\right)$ is bounded because of (2.2) in Proposition 2.1. Since for each fixed $z \in \mathbb{B}$, and using (2.1) in Proposition 2.1

$$
\lim _{m \rightarrow \infty}\left\|\left(I-L_{m}^{*}\right)\left(\delta_{z}\right)\right\|_{E^{*}}=\lim _{m \rightarrow \infty} \sup _{\|f\|_{E} \leq 1}\left|\left(I-L_{m}\right)(f)(z)\right|=0,
$$

the sequence $\left(I-L_{m}^{*}\right)_{m \in \mathbb{N}}$ converges to zero on every point in the total set $\left\{\delta_{z}: z \in \mathbb{B}\right\} \subset E^{*}$ as $m \rightarrow \infty$. So we appeal to the Banach-Steinhaus type theorem [15, III 4.5] to conclude that $\left(I-L_{m}^{*}\right)_{m \in \mathbb{N}}$ converges to zero uniformly on compact subsets of $E^{*}$. In particular on the image $\left\{T^{*}\left(\delta_{z}\right):|z| \leq t\right\} \subset E^{*}$ of the compact set $\left\{\delta_{z}:|z| \leq t\right\} \subset G_{v}^{\infty}$, that is,

$$
0=\lim _{m \rightarrow \infty} \sup _{\{|z| \leq t\}}\left\|\left(I-L_{m}^{*}\right)\left(T^{*}\left(\delta_{z}\right)\right)\right\|_{E^{*}}=\lim _{m \rightarrow \infty} \sup _{\left\{\|f\|_{E} \leq 1\right\}} \sup _{\{|z| \leq t\}}\left|\left\langle\left(I-L_{m}^{*}\right) \circ T^{*}\left(\delta_{z}\right), f\right\rangle\right| .
$$

Therefore since the weight $\widetilde{v}$ is bounded,

$$
\lim _{m \rightarrow \infty} \sup _{\left\{\|f\|_{E} \leq 1\right\}} \sup _{\{|z| \leq t\}}\left|\left[T \circ\left(I-L_{m}\right)\right](f)(z)\right| \widetilde{v}(z)=0 .
$$

On the other hand, for $|z|>t$, and as in the proof of [3, Theorem 3.1]

$$
\sup _{\left\{\|f\|_{E} \leq 1\right\}}\left|\left[T \circ\left(I-L_{m}\right)\right](f)(z)\right| \widetilde{v}(z) \leq\left\|I-L_{m}\right\|_{E \rightarrow H_{v}^{0}} \cdot \sup _{|z|>t} \frac{\left\|T^{*}\left(\delta_{z}\right)\right\|_{E^{*}}}{\left\|\delta_{z}\right\|_{v}} .
$$


Therefore,

$$
\begin{aligned}
\limsup _{m \rightarrow \infty}\left\|T-T \circ L_{m}\right\|_{E \rightarrow H_{v}^{0}} & =\limsup _{m \rightarrow \infty} \sup _{\left\{\|f\|_{E} \leq 1\right\}\{|z|<1\}} \sup _{m}\left|\left[T \circ\left(I-L_{m}\right)\right](f)(z)\right| \widetilde{v}(z) \\
& =\limsup _{m \rightarrow \infty} \sup _{\left\{\|f\|_{E} \leq 1\right\}\{|z|>t\}} \sup _{\mid}\left|\left[T \circ\left(I-L_{m}\right)\right](f)(z)\right| \tilde{v}(z) \\
& \leq \limsup _{m \rightarrow \infty}\left\|I-L_{m}\right\|_{E \rightarrow H_{v}^{0}} \cdot \sup _{|z|>t} \frac{\left\|T^{*}\left(\delta_{z}\right)\right\|_{E^{*}}}{\left\|\delta_{z}\right\|_{v}} .
\end{aligned}
$$

Applying Proposition 2.1 in (2.35) and letting $t \rightarrow 1$ we obtain $\|T\|_{e} \leq \ell$.

Corollary 2.6. Assume that $E$ satisfies conditions (C1)-(C6). If $u C_{\varphi}: E \rightarrow H_{v}^{0}$ is a bounded weighted composition operator, then

$$
\left\|u C_{\varphi}\right\|_{e}=\limsup _{|z| \rightarrow 1^{-}}|u(z)| \tilde{v}(z)\left\|\delta_{\varphi(z)}\right\|_{E^{*}}
$$

Proof. We apply Theorem 2.5 and just use that $\left(u C_{\varphi}\right)^{*}\left(\delta_{z}\right)=u(z) \delta_{\varphi(z)}$ and that $\left\|\delta_{z}\right\|_{v}=$ $1 / \widetilde{v}(z)$.

Example 2.7. If $E=H_{w}^{0}$, then from Corollary 2.6 we get the following extension of the onedimensional result in [5, Theorem 2.2]

$$
\left\|u C_{\varphi}\right\|_{e}=\limsup _{|z| \rightarrow 1^{-}} \frac{\widetilde{v}(z)|u(z)|}{\widetilde{w}(\varphi(z))}
$$

Corollary 2.8. Let $F$ be a Banach space of analytic functions in $\mathbb{B}$ that is isometrically isomorphic to the bidual of a space E satisfying conditions (C1)-(C6). Assume that E has the 1 -metric approximation property or is a dual space. Let $T: F \rightarrow H_{v}^{\infty}$ be a bounded operator such that $T(E) \subseteq H_{v}^{0}$ and whose restrictions to bounded subsets of $F$ are $\tau$ - $\tau$ continuous. Then

$$
\|T\|_{e} \simeq \limsup _{|z| \rightarrow 1^{-}} \frac{\left\|T^{*}\left(\delta_{z}\right)\right\|_{F^{*}}}{\left\|\delta_{z}\right\|_{v}}
$$

Proof. To see that $T=\left(T_{\left.\right|_{E}}\right)^{* *}$, it suffices to remark that $T$ is weak*-weak* continuous, that is, to check that $l \circ T \in E^{*}$ for all $l \in G_{v}^{\infty}$. And for this, it is enough to check that $l \circ T$ is weak* continuous on the closed unit ball $B_{E^{* *}}$ of $F=E^{* *}$, a fact that follows from observing that on $\left(B_{F}, w^{*}\right)$ the weak*-topology coincides with the Hausdorff coarser one $\tau$, for which both $T$ and $l$ are also continuous on bounded sets.

Since $E$ has the $\lambda$-metric approximation property or is a dual space, we may use Axler et al. [16] to obtain that $\|T\|_{e}=\left\|\left(T_{\mid E}\right)^{*}\right\|_{e} \simeq\left\|T_{\mid E}\right\|_{e}$. From this and Theorem 2.5 the corollary follows.

Notice that spaces like $H_{v}^{\infty}$, the Bloch space and BMOA satisfy the assumptions on $F$ in the above corollary. 
Corollary 2.9. Let $\beta>-1, \gamma>0$ and assume that $T: A_{\beta}^{1} \rightarrow A_{\beta}^{1}$ is a bounded operator. If $T^{\prime}\left(H_{\gamma}^{0}\right) \subseteq$ $H_{\gamma}^{0}$, where $T^{\prime}: H_{\gamma}^{\infty} \rightarrow H_{\gamma}^{\infty}$ is the dual operator with respect to the duality $\langle\cdot, \cdot\rangle_{\beta, \gamma}$, then

$$
\|T\|_{e} \simeq \limsup _{|z| \rightarrow 1^{-}} \frac{\left\|T\left(K_{z}^{\beta+\gamma}\right)\right\|_{A_{\beta}^{1}}}{\left\|K_{z}^{\beta+\gamma}\right\|_{A_{\beta}^{1}}} .
$$

Proof. Note that $\left\|K_{z}^{\beta+\gamma}\right\|_{A_{\beta}^{1}} \simeq\left(1-|z|^{2}\right)^{-\gamma}$. Let $T^{\prime}{ }_{\mid H_{\gamma}^{0}}$ be the restriction to $H_{\gamma}^{0}$ of $T^{\prime}$. Then we can apply Theorem 2.5 so that

$$
\left\|T^{\prime} H_{r}^{0}\right\|_{e}=\limsup _{|z| \rightarrow 1^{-}} \frac{\left\|\left(T^{\prime}{ }_{\mid H_{r}^{0}}\right)^{*}\left(\delta_{z}\right)\right\|_{\left(H_{r}^{0}\right)^{*}}}{\left\|\delta_{z}\right\|_{v_{\gamma}}} .
$$

For $\|f\|_{H_{r}^{\infty}} \leq 1, f \in H_{\gamma}^{0}$ and $z \in \mathbb{D}$,

$$
\left|\left(T^{\prime}{ }_{\mid H_{\gamma}^{0}}\right)^{*} \delta_{z}(f)\right|=\left|T^{\prime} f(z)\right|=\left|\left\langle K_{z}^{\beta+\gamma}, T^{\prime} f\right\rangle_{\beta, \gamma}\right|=\left|\left\langle T\left(K_{z}^{\beta+\gamma}\right), f\right\rangle_{\beta, \gamma}\right| .
$$

Since by [12] $H_{r}^{0}$ is isomorphic to $c_{0}$, we obtain that

$$
\|T\|_{e} \simeq\left\|\left(T_{\mid H_{r}^{0}}^{\prime}\right)^{*}\right\|_{e} \simeq\left\|T^{\prime} H_{r}^{0}\right\|_{e}
$$

as wanted.

Now we apply Theorem 2.5 to bounded operators on the subspaces of $p$-Bloch and little $p$-Bloch spaces consisting of all $f$ such that $f(0)=0$. We denote the spaces by $\mathbb{B}^{p} / \mathbb{C}$ and $\mathbb{B}_{0}^{p} / \mathbb{C}$ correspondingly. Similar subspaces of $H_{\mu}^{\infty}$ and $H_{\mu}^{0}$ are denoted by $H_{\mu}^{\infty} / \mathbb{C}$ and $H_{\mu}^{0} / \mathbb{C}$ correspondingly.

Observe that for any $z \in \mathbb{B},\left\|\delta_{z}\right\|_{\left(\mathcal{B}_{0}^{p}\right)^{*}} \leq 1+\left\|\delta_{z}\right\|_{\left(\mathcal{B}_{0}^{p} / \mathbb{C}\right)^{*}}$. Hence $\mathbb{B}_{0}^{p} / \mathbb{C}$ being a subspace of $\mathbb{B}_{0}^{p}$ fulfills conditions $(C 1)-(C 6)$.

Set $\widehat{\delta}_{z}(f):=\left(1-|z|^{2}\right)^{p} \mathfrak{R} f(z)$ for $z \in \mathbb{B}$ and $f \in \mathbb{B}_{p} / \mathbb{C}$.

Corollary 2.10. (a) Let $T: \mathbb{B}_{0}^{p} / \mathbb{C} \rightarrow \mathbb{B}_{0}^{p} / \mathbb{C}$ be a bounded operator. Then

$$
\|T\|_{e}=\limsup _{|z| \rightarrow 1^{-}}\left\|T^{*}\left(\widehat{\delta}_{z}\right)\right\|_{\left(\mathcal{B}_{0}^{p} / \mathbb{C}\right)^{*}}
$$

(b) Let $T: \mathbb{B}^{p} / \mathbb{C} \rightarrow \mathbb{B}^{p} / \mathbb{C}$ be a bounded operator such that $\mathbb{B}_{0}^{p} / \mathbb{C}$ is an invariant subspace for $T$ and whose restrictions to the bounded subsets are $\tau-\tau$ continuous. Then

$$
\|T\|_{e} \simeq \limsup _{|z| \rightarrow 1^{-}}\left\|T^{*}\left(\widehat{\delta}_{z}\right)\right\|_{\left(\mathbb{B}^{p} / \mathbb{C}\right)^{*}}
$$


Proof. Let $\mathfrak{R}: \mathbb{B}^{p} / \mathbb{C} \rightarrow H_{p}^{\infty} / \mathbb{C}$ be defined by $\Re(f)(z)=\Re f(z)$. Then both $\mathfrak{R}$ and $\mathfrak{R}: \mathbb{B}_{0}^{p} / \mathbb{C} \rightarrow$ $H_{p}^{0} / \mathbb{C}$ are onto isometries (see, e.g., [17]). Therefore, if we put $L=\mathfrak{R} \circ T$, we have $\|L\|_{e}=\|T\|_{e}$. Since $\left\|\delta_{z}\right\|_{v_{p}}=\left(1-|z|^{2}\right)^{-p}$, and $\mathfrak{R}(f)(z)=\mathfrak{R}^{*}\left(\delta_{z}\right)(f)$ we also have that

$$
L^{*}\left(\frac{\delta_{z}}{\left\|\delta_{z}\right\|_{v_{p}}}\right)=T^{*} \circ \mathfrak{R}^{*}\left(\frac{\delta_{z}}{\left\|\delta_{z}\right\|_{v_{p}}}\right)=T^{*}\left(\left(1-|z|^{2}\right)^{p} \mathfrak{R}^{*}\left(\delta_{z}\right)\right)=T^{*}\left(\widehat{\delta}_{z}\right) .
$$

Hence, $\left\|T^{*}\left(\widehat{\delta}_{z}\right)\right\|_{\left(\mathcal{B}_{0}^{p} / \mathbb{C}\right)^{*}}=\left\|L^{*}\left(\delta_{z} /\left\|\delta_{z}\right\|_{v}\right)\right\|_{\left(\mathbb{B}_{0}^{p} / \mathbb{C}\right)^{*}}$.

Now for (a), apply Theorem 2.5 to $L$. For (b), observe that $L\left(\mathbb{B}_{0}^{p} / \mathbb{C}\right) \subseteq H_{p}^{0}$ and also that the quotient norm on $\mathbb{B}_{0}^{p} / \mathbb{C}$ coincides with $b_{p}$, and therefore using the quotient and the subspace duality, $\left(\mathbb{B}_{0}^{p} / \mathbb{C}\right)^{* *}=\mathbb{B}^{p} / \mathbb{C}$. Moreover, $L$ is $\tau-\tau$ continuous on bounded sets, because $\mathfrak{R}$ preserves the $\tau$ - $\tau$ continuity. Then use Corollary 2.8 .

For a given $g \in H(\mathbb{B}), g(0)=0$ and a holomorphic self-map $\varphi$ of $\mathbb{B}$, the following integral-type operator on $H(\mathbb{B})$ is introduced in [17]

$$
P_{\varphi}^{g}(f)(z)=\int_{0}^{1} f(\varphi(t z)) g(t z) \frac{d t}{t}, \quad f \in H(\mathbb{B}), z \in \mathbb{B} .
$$

This operator has also been studied later, for example, in $[6,8,18,19]$. For a closely related operator see also [20]. In [6] Stević calculated the essential norm of the operator $P_{\varphi}^{g}$ : $A_{\beta}^{2} \rightarrow B_{\mu}$. Motivated by this result we calculate here the essential norm of the operator $P_{\varphi}^{g}$ : $A_{\beta}^{p} \rightarrow B_{\mu, 0} / \mathbb{C}$, but in terms of the associated weight of $\mu$.

Corollary 2.11. Assume that $\beta>-1, p>1, g \in H(\mathbb{B}), g(0)=0, \varphi$ is a holomorphic self-map of $\mathbb{B}$ and $P_{\varphi}^{g}: A_{\beta}^{p} \rightarrow \mathbb{B}_{\mu, 0} / \mathbb{C}$ is bounded. Then

$$
\left\|P_{\varphi}^{g}\right\|_{e}=\limsup _{|z| \rightarrow 1} \frac{\tilde{\mu}(z)|g(z)|}{\left(1-|\varphi(z)|^{2}\right)^{(n+1+\beta) / p}} .
$$

Proof. Since $\mathfrak{R} P_{\varphi}^{g}(f)(z)=f(\varphi(z)) g(z)$, we have $\mathfrak{R} P_{\varphi}^{g}=g C_{\varphi}: A_{\beta}^{p} \rightarrow H_{\mu}^{0} / \mathbb{C}$. From this and the fact that the operator $\Re$ is an onto isometry between $\mathbb{B}_{\mu, 0} / \mathbb{C}$ and $H_{\mu}^{0} / \mathbb{C}$ it follows that $\left\|P_{\varphi}^{g}\right\|_{e}=\left\|g C_{\varphi}\right\|_{e}$. By Corollary 2.6, we have

$$
\left\|g C_{\varphi}\right\|_{e}=\limsup _{|z| \rightarrow 1^{-}}|g(z)| \tilde{\mu}(z)\left\|\delta_{\varphi(z)}\right\|_{A_{\beta}^{q}}
$$

where $1 / p+1 / q=1$. Therefore equality (2.47) follows simply bearing in mind (2.24).

\section{Acknowledgments}

P. Galindo is supported partially by Project MEC-FEDER 2007 064521. M. Lindström is supported partially by Magnus Ehrnrooths stiftelse and Project MEC-FEDER 2007064521. 
S. Stević is supported partially by the Serbian Ministry of Education and Science (projects III44006 and III41025).

\section{References}

[1] J. Bonet, P. Domański, and M. Lindström, "Essential norm and weak compactness of composition operators on weighted Banach spaces of analytic functions," Canadian Mathematical Bulletin, vol. 42, no. 2, pp. 139-148, 1999.

[2] C. C. Cowen and B. D. MacCluer, Composition Operators on Spaces of Analytic Functions, Studies in Advanced Mathematics, CRC Press, Boca Raton, FL, USA, 1995.

[3] P. Galindo and M. Lindström, "Essential norm of operators on weighted Bergman spaces of infinite order," Journal of Operator Theory, vol. 64, no. 2, pp. 387-399, 2010.

[4] M. Lindström and E. Wolf, "Essential norm of the difference of weighted composition operators," Monatshefte für Mathematik, vol. 153, no. 2, pp. 133-143, 2008.

[5] A. Montes-Rodríguez, "Weighted composition operators on weighted Banach spaces of analytic functions," Journal of the London Mathematical Society. Second Series, vol. 61, no. 3, pp. 872-884, 2000.

[6] S. Stević, "Essential norm of an operator from the weighted Hilbert-Bergman space to the Bloch-type space," Ars Combinatoria, vol. 91, pp. 123-127, 2009.

[7] S. Stević, "Norm and essential norm of composition followed by differentiation from $\alpha$-Bloch spaces to $H_{\mu}^{\infty}$," Applied Mathematics and Computation, vol. 207, no. 1, pp. 225-229, 2009.

[8] S. Stević, "On a new integral-type operator from the Bloch space to Bloch-type spaces on the unit ball," Journal of Mathematical Analysis and Applications, vol. 354, no. 2, pp. 426-434, 2009.

[9] K. Zhu, Spaces of Holomorphic Functions in the Unit Ball, vol. 226 of Graduate Texts in Mathematics, Springer, New York, NY, USA, 2005.

[10] K. D. Bierstedt, J. Bonet, and J. Taskinen, "Associated weights and spaces of holomorphic functions," Studia Mathematica, vol. 127, no. 2, pp. 137-168, 1998.

[11] K. D. Bierstedt and W. H. Summers, "Biduals of weighted Banach spaces of analytic functions," Australian Mathematical Society. Series A, vol. 54, no. 1, pp. 70-79, 1993.

[12] W. Lusky and J. Taskinen, "On weighted spaces of holomorphic functions of several variables," Israel Journal of Mathematics, vol. 176, pp. 381-399, 2010.

[13] K. F. Ng, "On a theorem of Dixmier," Mathematica Scandinavica, vol. 29, pp. 279-280, 1971.

[14] A. L. Shields and D. L. Williams, "Bonded projections, duality, and multipliers in spaces of analytic functions," Transactions of the American Mathematical Society, vol. 162, pp. 287-302, 1971.

[15] H. H. Schaefer, Topological Vector Spaces, Springer, 1980.

[16] S. Axler, N. Jewell, and A. Shields, "The essential norm of an operator and its adjoint," Transactions of the American Mathematical Society, vol. 261, no. 1, pp. 159-167, 1980.

[17] S. Stević, "On a new operator from $H^{\infty}$ to the Bloch-type space on the unit ball," Utilitas Mathematica, vol. 77, pp. 257-263, 2008.

[18] S. Stević, "On an integral-type operator from logarithmic Bloch-type and mixed-norm spaces to Blochtype spaces," Nonlinear Analysis, vol. 71, no. 12, pp. 6323-6342, 2009.

[19] S. Stević, “On operator $P_{\varphi}^{g}$ from the logarithmic Bloch-type space to the mixed-norm space on the unit ball," Applied Mathematics and Computation, vol. 215, no. 12, pp. 4248-4255, 2010.

[20] S. Stević, "On an integral operator between Bloch-type spaces on the unit ball," Bulletin des Sciences Mathématiques, vol. 134, pp. 329-339, 2010. 


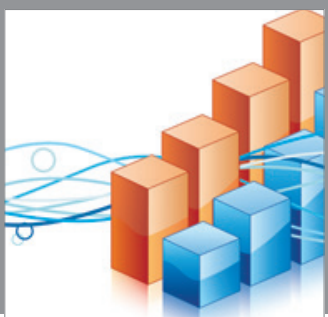

Advances in

Operations Research

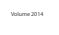

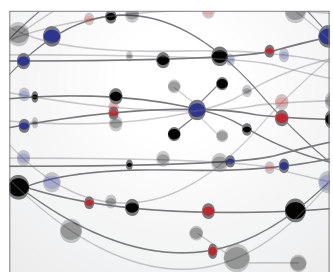

\section{The Scientific} World Journal
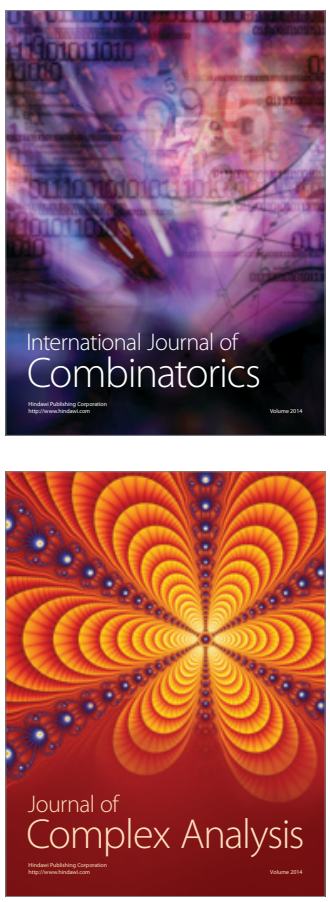

International Journal of

Mathematics and

Mathematical

Sciences
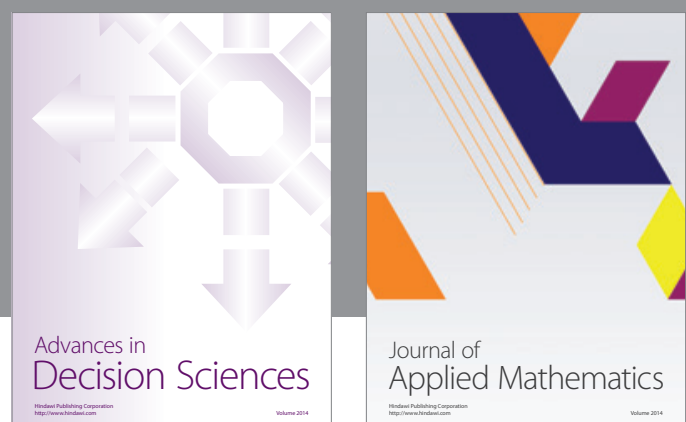

Journal of

Applied Mathematics
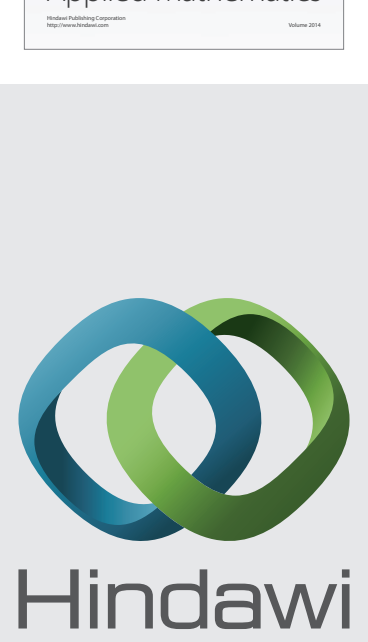

Submit your manuscripts at http://www.hindawi.com
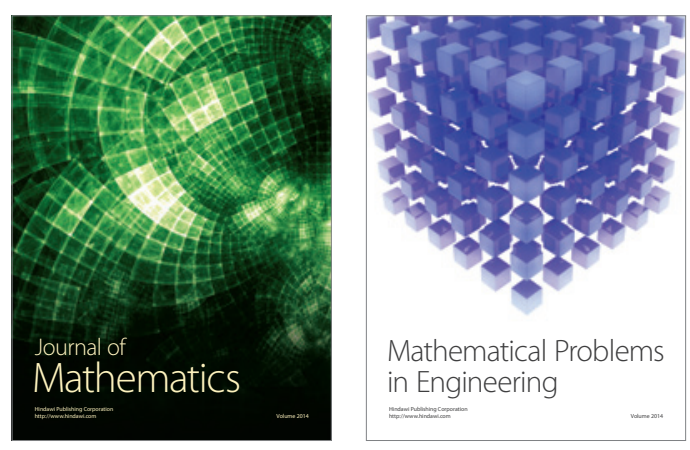

Mathematical Problems in Engineering
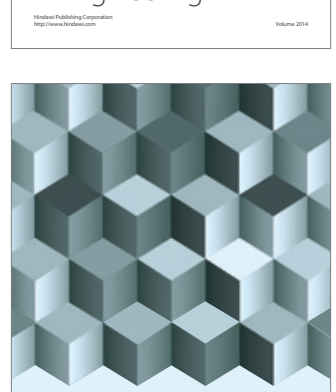

Journal of

Function Spaces
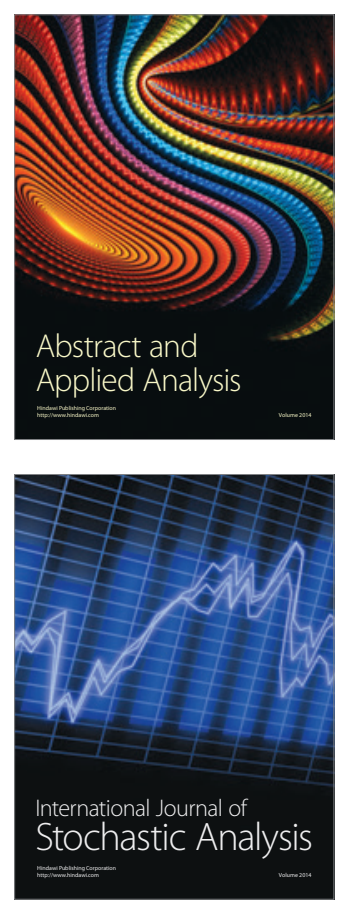

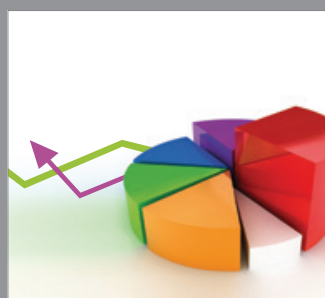

ournal of

Probability and Statistics

Promensencen
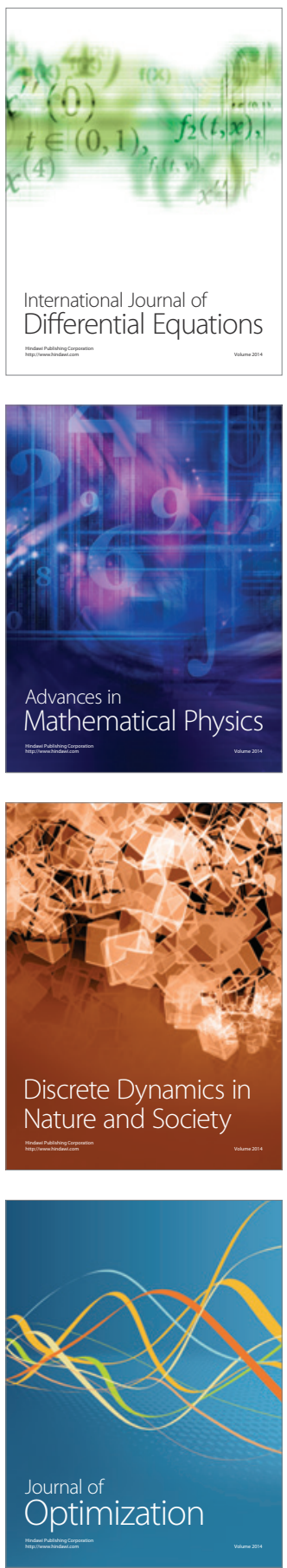